\title{
A new methodology and finding relating to visual stimulus selection in children'
}

TERRY T. FAW AND JUM C. NUNNALJ,Y, DEPARTMENT OF PSYCHOLOGY, VANDERBILT UNIVERSITY, Nashville, Tenn. 37203

The purposes of the present study were: (a) to examine more closely the effects of stimulus incongruity on visual selection in children by employing stimuli which represented four points on a continuum of incongruity; (b) to employ a procedure which would closely approximate a natural environment and thus increase the situational generality of the results. Results demonstrating the influence of stimulus incongruity on fixationdominance were replicated using a modified procedure in which a child sat in a waiting room unaware that his looking behavior was observed. This replication lends credance to the situational generality of those earlier findings. The extension of this basic replication indicates that with children the relationship between stimulus incongruity and fixation-dominance is monotonic and increasing.

Recent years have witnessed numerous attempts to investigate the stimulus characteristics and motivational variables which influence selective attention. One aspect of selective attention which has been investigated is that of fixation-dominance (FD), which refers to the tendency of $S s$ to fixate one part of a particular visual display longer than other parts.

In studies of FD to date, the stimulus characteristics examined most often have concerned various aspects of stimulus "information value," e.g., complexity and novelty. Here information value is considered an increasing function of the difficulty to encode (identify or classify) a stimulus. Evidence from previous research supports the hypotheses that FD is determined in part by the information value of a stimulus (Berlyne, 1958; Faw \& Nunnally, 1967,1968 ). In those studies more complex or novel stimuli were looked at longer than their less complex or novel counterparts.

Although the results from earlier studies are consistent among themselves and are in keeping with the hypothesis that FD is positively related to information value, most of those studies were characterized by two features which potentially limit the generality of findings. First, in most investigations, only two levels of the particular stimulus characteristic under study were compared, e.g., comparison of a novel picture with a banal picture. The use of only two points on the continuum representing the stimulus variable severely limits the extent to which one can examine the relationship between that variable and FD. This procedural difficulty is most evident in studies designed to examine the influence of stimulus novelty, as defined by Berlyne (1958) in terms of incongruity, on FD. One purpose of the present experiment was to examine more closely the relationship between stimulus incongruity and FD by employing stimuli which represented four distinct points on a continuum of incongruity.

A second problem characterizing most research on FD is that of "laboratory effects." In the typical study $S$ enters a sterile experimental environment and is required to push a button or make some other motor response in order to view various stimuli provided by $E$. Although these experimental settings provide $E$ with excellent control over S's environment and enable accurate and convenient measurement of looking behavior, the settings are only distantly related to real life environments. One wonders to what extent the findings from such artificial situations faithfully mirror the principles that apply to looking behavior of people walking down a street or sitting in an airline terminal. A second purpose of the present experiment was to attempt to employ a procedure and experimental setting which more closely represented the natural environment. In essence what was done was to surreptitiously measure the effects of different levels of stimulus incongruity on looking behavior while children sat in a comfortable waiting room. The children were sitting there for a plausible reason, and they had no cause to think that they were participating in an experiment on looking behavior. If results comparable to those obtained in earlier studies were obtained using these modified procedures, it would lend credance to the situational generality of those findings. Also, whether or not the procedures employed in this experiment led to similar findings as those in most previous research on FD, they certainly lend themselves to fewer embarrassing questions regarding "laboratory effects."

Subjects. The Ss were 11 boys and 14 girls from the third grade of a Metropolitan Nashville elementary school. Their ages ranged from 8 years, 5 months to 10 years, 1 month, with a mean age of 9 years, 1 month. They may be described as coming from a middle-class socio-economic background.

Stimuli. To increase the generality of results, two different sets of four stimuli were employed in the present study. Each set consisted of a banal stimulus and stimuli representing three additional levels of incongruity. The novelty levels of stimuli were determined prior to the experiment by having a separate group of 59 third- and fourth-grade children rank order the stimuli in each set from most familiar (1) to most unusual (4). The stimuli were achromatic line drawings produced by a commercial artist. A description of the stimuli in each set as well as their mean ranks follows.

Set 1: Normal man (1.20); man with short stubby legs and long arms (1.90); man with four arms (3.20); man with wings and a dog's head (3.70).

Set 2: Normal Holstein cow (1.04); cow with polka dots (2.27); cow with disproportionately long legs $(2.80)$; cow with long legs, the trunk of an elephant, and the tail of an airplane (3.90).

Apparatus. The experiment was conducted in a $22 \mathrm{ft} \times 7 \mathrm{ft} 7$ in. trailer which was partitioned into two rooms. The experimental room was $6 \mathrm{ft} 7$ in. $x 7 \mathrm{ft} 2$ in. and was panelled in natural wood. The floor was carpeted, and the room was furnished with two moulded plastic chairs, a coffee table, an end table, and a table lamp. On the wall separating the experimental room and observation room were two 21 in. $x 14$ in. rear projection screens mounted in picture frames. The inside edges of the screens were separated by 52 in. and their lower edge was $201 / 2$ in. from the floor. In the center of the wall, hanging 56 in. from the floor, was an 8 in. $\times 10$ in. one-way viewing mirror, through which an observer $(O)$ could view the behavior of $S$.

The observation room was isolated from the experimental room. It contained slide projectors which were used to project stimulus slides onto the rear of the viewing screens. The projectors were attached to a timing device which automatically advanced the projectors every $15 \mathrm{sec}$. From the observation room, $O$ could view the behavior of $S$ and record whether $S$ was looking at the left or right viewing screen. Recording was accomplished by depressing either of two buttons which activated pens on a continuous tape event recorder.

Procedure. Prior to the experiment, Ss were seen as a group and told that $\mathrm{E}$ was attempting to discover the kinds of toys children enjoyed. They were told that each $S$ would receive $25 \notin$ for participating in the study. Ss were then brought individually to the experimental trailer. Shortly before $S$ and $E$ entered the experimental room, $O$ activated the timing apparatus and projectors which began to project pictures onto the viewing screen. Thus, the changing pictures on the viewing screens were a part of the environment into which $\mathbf{S}$ entered. After obtaining biographical information from $S$ and familiarizing him with the experimental room, E said, "I have a lot of toys to ask you about, but I need to gather them together before we can continue. While I arrange the toys, you can wait here in the trailer. If you would like, you can look at the pictures on our picture screens. I will be gone just a few minutes." Shortly after E's departure, the stimulus slides representing comparisons of more and less incongruent stimuli 


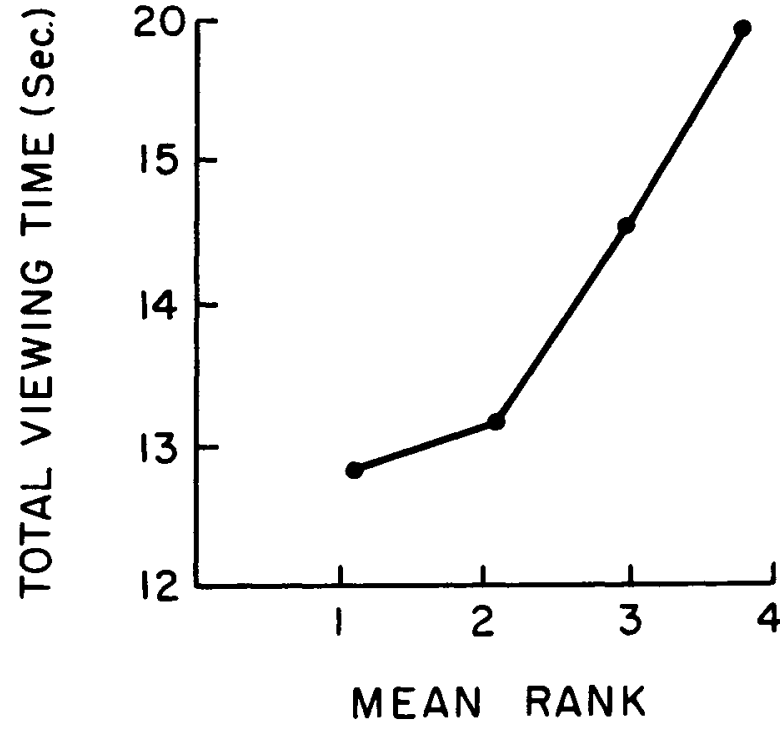

Fig. 1. Mean total looking time toward stimuli representing four levels of incongruity. (Results from two sets of stimuli are combined. Mean ranks were obtained by averaging the mean ranks of the two stimuli which represented similar levels of incongruity.)

were projected onto the viewing screens. The six possible pairs of stimuli within each of the two sets were projected onto the viewing screens. The order of presentation of 12 pairs of stimuli was determined randomly. The left-right position of the stimulus in each pair was reversed for 12 of the 25 Ss. Unknown to $S, O$ recorded which viewing screen was being viewed by $S$. No record was made of fixations directed at objects other than the viewing screens. Postexperimental questioning of Ss substantiated that they were unaware of $O$ 's presence.

Shortly after the 12 pairs of slides had been shown, E returned to the experimental room with a number of toys and games. $E$ continued asking $S$ questions regarding his preference for the various toys and finally terminated the experiment by paying $S$ $25 \$$.

Results. Measures of five potentially useful characteristics of S's viewing behavior were obtained from the record of his visual fixations. For each stimulus in each pair, the following information was recorded: (a) total viewing time, (b) length of longest fixation, (c) length of first fixation, (d) number of fixations directed toward the stimulus, and (e) mean length of fixations (i.e., total fixation time divided by number of fixations). Although each stimulus appeared in three different pairs during the course of the experiment, a single score was obtained for each stimulus on each measure by summing the values obtained on each of the three presentations.

The results from the five separate measures were each analyzed using a Ss by A (two sets of stimuli) by B (four levels of incongruity) analysis of variance. No significant effects were found in those analyses involving, either number of fixations or mean length of a fixation. The results of the other three measures were similar. To avoid repetition, only the results from total louking time are reported. The initial analysis of variance revealed only a significant main effect due to level of incongruity: $F(3,72)=$ $3.736, p<.02$. A subsequent trend analysis revealed a significant linear trend: $F(1,72)=10.377, p<.01$. The linear relationship between incongruity and total looking time is represented in Fig. 1. That figure clearly shows the increasing monotonic relationship between stimulus incongruity and FD.

To examine the possible change in looking behavior as a function of continued exposure to pairs of incongruent stimuli, the amount of time spent viewing either picture in a pair as opposed to other objects in the room was determined separately for the pair of pictures in each of the 12 positions of the experimental series. In addition, the percentage of time spent viewing the more incongruent stimulus in each pair was computed. Analysis of variance and subsequent post hoc comparisons revealed that the time spent viewing the test stimuli decreased significantly during the second half of the 12 pair series $(p<.01)$. However, when one examines the percentage of time spent viewing the more incongruent stimulus in the pair, regardless of the levels of incongruity being compared, no significant change is noted as a function of the pair's position in the sequence.

Discussion. The results of the present study are in keeping with the hypothesized increasing monotonic relationship between stimulus incongruity and FD. Not only are the results from the present study in the same direction as those reported earlier, but the percentage of time spent looking at the more incongruent stimulus in each pair is of the same magnitude as that reported in earlier studies (Faw \& Nunnally, 1968). Thus, the present study suggests that, in spite of the artificial atmosphere in which earlier studies were undertaken, the findings of those earlier experiments reflect the visual stimulus selection behavior of Ss in more natural environments.

The present study concerned only one stimulus characteristic, novelty in the form of incongruity. It may be that investigations of other stimulus variables (e.g., rated pleasantness of pictures) will produce different results in more naturalistic settings rather than in more laboratory-like settings. Regardless of the similarities and differences between results obtained in these two types of settings, the more naturalistic setting logically is a better place in which to investigate the determinants of looking behavior, because it is subject to so many fewer embarrassing questions about "external validity."

\section{REFERENCES}

BERLYNE, D. D. The influence of complexity and novelty in visual figures on orienting responses. J. exp. Psychol., 1958, 55, 289-296.

FAW, T. T., \& NUNNALLY, J. C. The effects on eye movements of complexity, novelty, and affective tone. Percept. \& Psychophys., 1967, 2, 263-267.

FAW, T. T., \& NUNNALLY, J. C. The influence of stimulus complexity, novelty, and affective value on children's visual fixations. $J$. exp. Child Psychol., 1968, 6, 141-153.

\section{NOTE}

1. While this research was in progress the first author was receiving support from a National Defense Graduate Fellowship. The study was supported, in part, by research Grant No. HD-03083, from the National Institute of Child Health and Human Development.

We wish to thank Adrienne Barnard for her invaluable assistance in the execution of this experiment. Appreciation is also expressed to the Metropolitan School System of Nashville, Tennessee and, in particular, to the principals and teachers of Glenview and Cockrill elementary schools for their cooperation in making $S s$ available for the research. 